\title{
Ultrahigh frequency microplasmas from 1 pascal to 1 atmosphere $\dagger$
}

\author{
J. Hopwood and F. Iza
}

Northeastern University, Boston, Massachusetts 02115, USA

Received 5th March 2004, Accepted 30th April 2004

First published as an Advance Article on the web 5th August 2004

\begin{abstract}
The generation of plasma-on-a-chip is examined for two extremes in gas pressure. The application of microplasmas as sensors of industrial vacuum processes requires stable operation at gas pressures of less than $1 \mathrm{~Pa}$. In this low-pressure regime, the addition of a static magnetic field that causes electron cyclotron resonance is shown to increase the emission intensity of the microplasma by $50 \%$. Using atomic emission spectrometry, the detection of helium in air is found to have a detection limit of $1000 \mathrm{ppm}$, which is three orders of magnitude worse than the $\mathrm{DL}$ of $\mathrm{SO}_{2}$ in argon. The loss of sensitivity is traced to the high excitation energy threshold of $\mathrm{He}$, and to the poor ionization efficiency inherent in an air plasma. At atmospheric pressure $\left(10^{5} \mathrm{~Pa}\right)$, a microdischarge is described that operates in a $25 \mu \mathrm{m}$-wide gap in a microstrip transmission line resonator operating at $900 \mathrm{MHz}$. The volume of the discharge is $\sim 10^{-7} \mathrm{~cm}^{3}$, and this allows an atmospheric air discharge to be initiated and sustained using less than $3 \mathrm{~W}$ of power.
\end{abstract}

\section{Introduction}

The miniaturization of devices used for atomic emission spectrometry (AES) of gaseous discharges has recently garnered attention due to the promise of inexpensive, portable chemical analysis systems. The central component of a miniature AES system is a small, efficient plasma, sometimes referred to as a microplasma or a plasma-on-a-chip. There are two broad classes of applications for this technology. The first application requires a microplasma that operates at or near atmospheric pressure. The attractive feature of the atmospheric pressure microplasma is the elimination of large and expensive vacuum pumps. If one could also generate a microplasma in air, then the need for bulky cylinders of inert gases would be eliminated. Through direct sampling of atmospheric air by a microplasma, one can envision low-cost environmental monitors based on AES. A second application for miniaturized AES is the monitoring of industrial processes occurring at reduced pressure. The microelectronics industry, for example, relies heavily on vacuum processing to manufacture integrated circuits. These precise processes require continuous monitoring, and microplasma-based AES is a promising technology for vacuum process control. These two examples establish the need for microplasmas operating from less than $1 \mathrm{~Pa}$ up to $10^{5} \mathrm{~Pa}$ (1 atm). It is relatively easy to generate a microplasma in the pressure regime between $50 \mathrm{~Pa}$ and $5 \mathrm{kPa}$, but operation at extreme pressures requires more detailed attention to plasma physics and generator design. This paper investigates both low and high pressure microplasmas.

The primary technical issues of microplasma generation parallel those of large-scale gaseous discharges. In the case of a microplasma, however, the importance of three phenomena is amplified due to the practical constraints imposed by microsystems. First, unintentional ion-induced erosion of the plasma generator's microstructure will have a deleterious effect on the repeatability of emission spectra and the lifetime of the microplasma generator. It is possible to reduce ion erosion by using an ultra-high frequency (UHF) or microwave frequency power source. Second, unlike plasma torches, any heat generated by the microplasma must be minimized. Lastly, the ionization efficiency of the plasma must be optimized to

$\dagger$ Presented at the 2004 Winter Conference on Plasma Spectrochemistry, Fort Lauderdale, FL, USA, January 5-10, 2004. minimize power consumption by the microsystem. Power is a critical parameter that affects both the cost of the system's electronics and the minimum battery capacity required for portable applications. Fig. 1 illustrates the partitioning of power within a plasma and shows how the plasma properties described above are related.

Power is first partitioned between the plasma and the apparatus that supports the plasma, as shown in Fig. 1. The loss of power within the generator may be due to inefficiency in the electronics, parasitic electrical heating of the microstructure, or electromagnetic radiation. The remainder of the power is absorbed by free electrons in the microplasma. At the next level shown in Fig. 1 the power absorbed by free electrons in the plasma is distributed through collisions. The partitioning of power into ionizing collisions is necessary for replacing lost electrons, thereby sustaining the discharge. The power that is used for atomic excitation is also beneficial for applications involving emission spectrometry. The remaining paths, however, should be minimized within the microplasma. Electrons and ions that are accelerated toward the walls of the plasma vessel, or toward the electrodes, will generate heat and sputter erosion. Dissipation of power at the walls and electrodes is not only wasteful, it is also destructive. The use of low voltage, high frequency power supplies reduces wall losses within the microplasma. Low voltage operation prevents the excessive acceleration of ions and electrons toward the electrodes and ensures that the plasma potential is also low. Dissociation, and

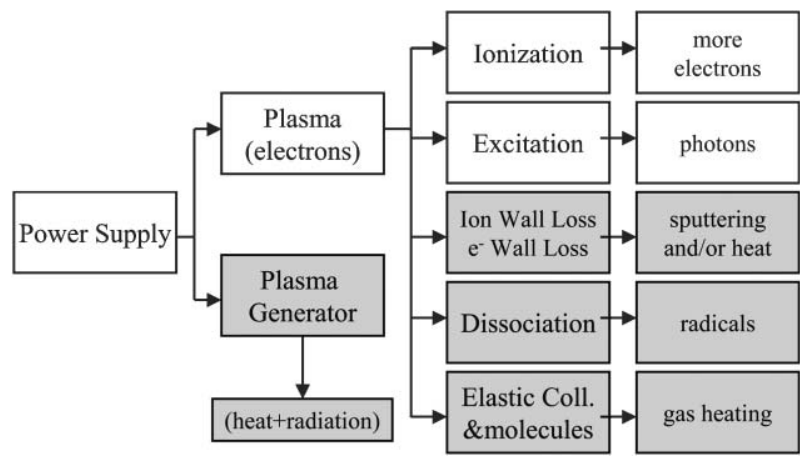

Fig. 1 This chart shows the various mechanisms for power loss within a plasma. In general, the shaded boxes represent undesirable power loss. 
its related power loss, is necessary for atomic emission spectrometry of molecular species. In an air discharge, however, dissociation of $\mathrm{N}_{2}$ and $\mathrm{O}_{2}$ consumes a large fraction of the total available power and results in significantly reduced ionization and light emission. Finally, low energy electrons transfer power to the gas by elastic collisions as well as excitation of vibrational and rotational molecular levels. This energy transfer path results in the heating of the plasma gas. In an atmospheric pressure ICP, the transfer of electron energy to the gas results in plasma temperatures exceeding $5000 \mathrm{~K}$. In a miniature plasma source, however, such high temperatures damage the microstructure and are generally avoided.

The interest in microplasma generation has resulted in a large number of publications in the past five years. A comprehensive review may be found in reference 1. Although it is impractical to cite all of the relevant work, a few illustrative examples are given. Microplasmas operating from a high dc voltage ${ }^{2-4}$ are simple, but the high cathode voltage results in ion-induced erosion. This erosion, however, can be used to detect metals in solution by using microfabricated liquid electrodes ${ }^{5,6}$ in place of metal electrodes. Cathodes with a hollow geometry reduce the discharge voltage and extend the useful lifetime of the microplasma. ${ }^{7,8}$ Dielectric barrier discharges (DBD) are also the subject of intense research for their simplicity and extensive use in plasma displays. ${ }^{9,10}$ In addition, at higher frequencies of operation the plasma electrodes may be covered with a protective dielectric layer that further extends the reliability of the device. Sources operating at radio frequencies have also been investigated. Examples include the capacitively coupled plasma source, ${ }^{11,12}$ the plasma needle, ${ }^{13}$ and the miniature ICP. ${ }^{14}$ Finally, plasma sources operating at microwave frequencies and atmospheric pressure have been miniaturized. ${ }^{15-17}$ Of particular note is that the plasma potential of both the miniature ICP and microwave plasmas is less than the threshold for sputtering. This allows UHF and microwave plasmas to operate with high ionization efficiency and little or no sputter erosion.

Very little work has been reported on microplasma operation at low pressure. In general, the product of gas pressure $(p)$ and plasma size $(d)$ should remain approximately constant, i.e., $p d \sim 100 \mathrm{~Pa} \mathrm{~cm}$. This fundamental scaling law of discharges makes the operation of miniature plasmas $(d<1 \mathrm{~cm})$ difficult at low pressure $(p \ll 100 \mathrm{~Pa})$. Electrons readily diffuse to the walls at low pressure, making the loss rate of electrons unacceptably high in a low-pressure microplasma. In addition to the loss of electrons, the mean free path for ionization will also exceed the size of the plasma. Lastly, the coupling of power to electrons is most effective if the electron collision frequency equals the power supply frequency. ${ }^{18}$ As a rule of thumb, the ideal pressure for power coupling to electrons is $p \approx 1 / 4 f$ where $p$ is the pressure in $\mathrm{Pa}$ and $f$ is the power supply frequency in $\mathrm{MHz}$. At pressures far below the optimum $(p \ll 1 / 4 f)$, it becomes difficult to transfer power to the electrons and the plasma generator becomes inefficient (Fig. 1). To operate at lower pressures, it is common practice to permeate the plasma with a static magnetic field. Through the Lorentz force, the magnetic field traps electrons by inducing helical trajectories. The frequency of electron motion, or gyrofrequency, is given by $\omega=q B / m_{\mathrm{e}}$, where $B$ is the magnetic field strength and $m_{\mathrm{e}}$ is the mass of the electron. If $B$ is adjusted such that the power supply frequency matches the gyrofrequency $\left(2 \pi f=q B / m_{\mathrm{e}}\right)$, power is coupled directly to electrons ${ }^{18}$ in the absence of collisions through electron cyclotron resonance (ECR). ECR will be shown to improve the low pressure emission intensity of a miniature ICP.

Finally, we report the creation of a microplasma in air. At atmospheric pressure, the electron collision frequency is always much greater than any practical power supply frequency, making the production of energetic electrons difficult. A microwave frequency gap-excited discharge with $p d \sim 250 \mathrm{~Pa} \mathrm{~cm}$ was found to self-ignite and sustain an air discharge, however. This requires the micromachining of a discharge gap of $d=25 \mu \mathrm{m}$.

\section{Experiment}

\subsection{Low pressure apparatus $(<1000 \mathrm{~Pa})$}

Low pressure experiments were performed using a microfabricated inductively coupled plasma (mICP) that was formed on a dielectric substrate. Two different configurations were used in this work. The first consists of a 3-turn planar spiral inductor $(d=5 \mathrm{~mm})$ and a matching network micromachined on a glass wafer. The device, described in detail elsewhere, ${ }^{19}$ operates at $490 \mathrm{MHz}$, and typically uses a gas flow of 2-200 ml per minute through a $0.17 \mathrm{~cm}^{3}$ chamber at a pressure of 13 to $1300 \mathrm{~Pa}$. For very low pressure work, it was desirable to use a second, larger mICP because the increase in $d$ allows for the reduction in $p$. This device consists of a 5-turn planar spiral coil $(d=10 \mathrm{~mm})$ and matching network fabricated on a printed circuit board. ${ }^{20}$ The printed circuit board is placed adjacent to a $1.2 \mathrm{~mm}$-thick glass window on a 1-1/3 Conflat vacuum flange. As shown in Fig. 2, the flange is attached to a plasma etcher with a $5001 \mathrm{~s}^{-1}$ turbo pump that is capable of high vacuum operation $\left(<10^{-5} \mathrm{~Pa}\right)$. The discharge is not confined along the axis of the flange, but a typical discharge volume is $3 \mathrm{~cm}^{3}$. The mICP is sandwiched between two spools of wire that create a static magnetic field with the field vector primarily pointing along the axis of the flange. The magnitude of the $B$-field is proportional to the current $(I)$ supplied by a $0.0-5.0$ ampère current source. At the vacuum window the field was measured by a gauss meter and found to be

$$
B \text { (gauss) }=60 I \text { (ampères) }
$$

This particular $\mathrm{mICP}$ was operated at a frequency of $220 \mathrm{MHz}$, so the onset of electron cyclotron resonance is expected to occur as $B$ is increased to 78 gauss, corresponding to $I=1.3 \mathrm{~A}$.

Optical emission was measured along the axis of both the $5-\mathrm{mm}$ and $10-\mathrm{mm}$ inductively coupled plasmas by an optical multichannel analyzer (OMA). It consists of an EG\&G 1024pixel silicon photodiode detector Model 1453A that is cooled to approximately $-40{ }^{\circ} \mathrm{C}$, and a diffraction grating spectrometer (Jarrel-Ash Model 82-479). The spectral resolution of this instrument is $1 \mathrm{~nm}$ FWHM using a $100 \mu \mathrm{m}$ entrance slit.

\subsection{High pressure apparatus $(>1000 \mathrm{~Pa})$}

Although a microfabricated inductively coupled plasma has been demonstrated to operate in argon at a pressure of $1 \mathrm{~atm}$, the power consumption was approximately $50 \mathrm{~W} .^{21}$ Experimentally, one finds that the ICP devices described above are best suited for low pressure operation. Microstrip transmission lines, however, have been used by Broekaert's group ${ }^{15,16}$ to create atmospheric argon microplasmas using approximately $10 \mathrm{~W}$. In this section, we describe a microstrip microwave plasma source that sustains an atmospheric pressure microplasma in room air. As is shown in Fig. 3, the plasma generator is made from a copper microstrip transmission line in the shape of a ring with a diameter of $20 \mathrm{~mm}$. The ring is microfabricated on a dielectric substrate with a relative permittivity of 10.2. The substrate is backed by a copper ground plane. Microwave power $(900 \mathrm{MHz}, 0-3 \mathrm{~W})$ is coupled to the ring through a subminiature type-A connector (SMA). The ring structure is onehalf wavelength in circumference and resonates at the frequency of the power supply. As previously described, this resonant structure produces a strong electric field in the region of the discharge gap because the voltage at the 


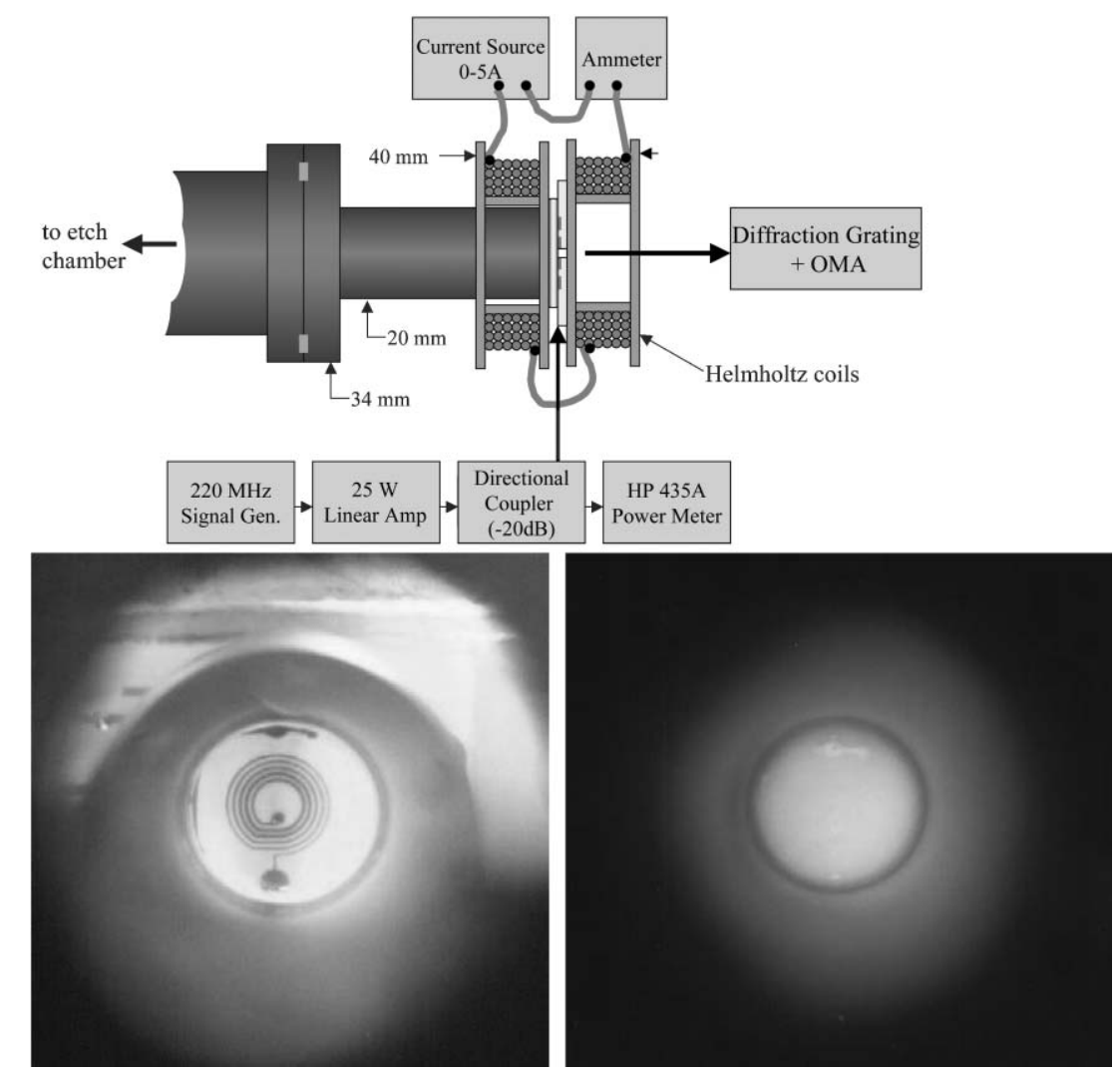

Fig. 2 A cross sectional schematic of the microfabricated ICP enhanced by a static magnetic field for low pressure operation. The two photographs show the mICP as viewed along the axis of the flange with the plasma off (left) and on (right).

two ends of the microstrip line is $180^{\circ}$ out of phase. ${ }^{22}$ Furthermore, the electric field in the discharge gap can be increased by making the gap smaller. In Fig. 3, the discharge gap is $25 \mu \mathrm{m}$ and the electric field is $>10^{6} \mathrm{~V} \mathrm{~m}^{-1}$. Unlike $\mathrm{dc}$ microplasma generators, however, no dc potential difference exists across the gap because the split-ring resonator's two electrodes are electrically connected through the microstrip transmission line. Because the operating frequency is greater than the ion-plasma frequency and the dc potential across the gap is zero, this microplasma generator eliminates ion erosion of the microfabricated electrodes.

\section{Experimental results}

\subsection{Operation in air}

A challenging spectroscopic problem is the detection of helium in room air. The difficulties include interference between $\mathrm{He}$ and the molecular spectrum of nitrogen, poor plasma intensity using air, quenching of excited states of $\mathrm{He}$ by oxygen and nitrogen, and the high excitation threshold energy of helium. In Fig. 4, a typical emission spectrum produced by an mICP from a $27 \mathrm{~Pa}$ air plasma is shown (top curve). Although the gas flow contains $1 \% \mathrm{He}$, the first positive band of $\mathrm{N}_{2}$ dominates the

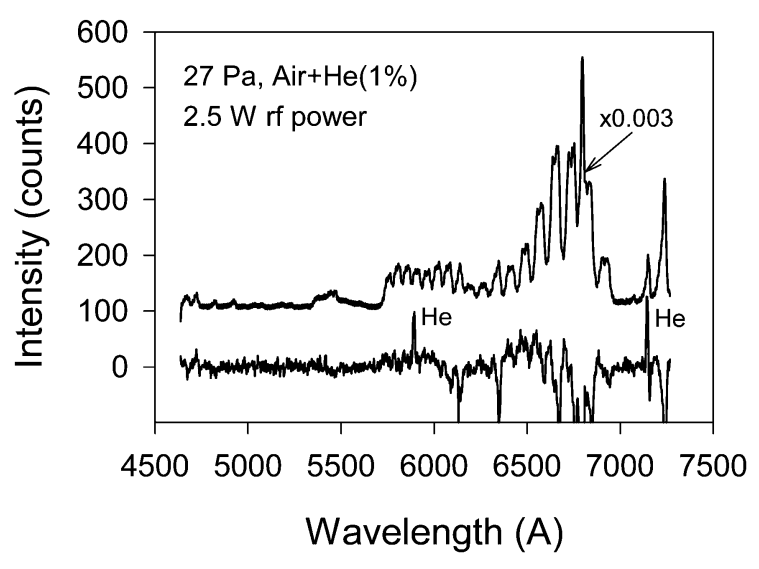

Fig. 4 The emission spectrum for an air plasma (27 Pa) with $1 \% \mathrm{He}$ (top) and the helium emission observed after subtraction of the background air spectrum (bottom).

spectrum and obscures He emission. The lower spectrum in Fig. 4 is obtained by subtracting the plasma emission, due to laboratory air, from the upper spectrum containing $1 \% \mathrm{He}$. Once the signal due to the air plasma is removed, one can

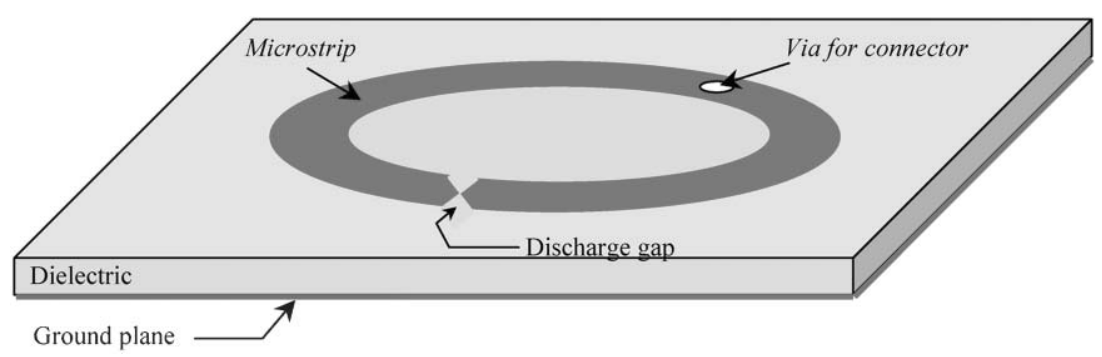

Fig. 3 A micromachined discharge gap $(d=25 \mu \mathrm{m})$ in this split-ring resonator creates a self-igniting microplasma in atmospheric pressure air. 
Table 1 Comparison of plasma factors that influence photon emission and detection limits

\begin{tabular}{|c|c|c|c|c|c|c|c|}
\hline & Pressure/Pa & Electron temperature/eV & $k_{\mathrm{ex}} / \mathrm{cm}^{3} \mathrm{~s}^{-1}$ & $E_{\mathrm{c}} / \mathrm{eV}$ per ion & $n_{\mathrm{e}} / \mathrm{cm}^{-3}$ & $k_{\mathrm{ex}} n_{\mathrm{e}} / \mathrm{s}^{-1}$ & $\mathrm{DL}(\mathrm{ppm})$ \\
\hline $\mathrm{SO}_{2}$ in $\mathrm{Ar}$ & 330 & 1.6 & $3.5 \times 10^{-10}$ & 120 & $5 \times 10^{16}$ & $1.7 \times 10^{7}$ & 0.2 \\
\hline $\mathrm{He}$ in air & 27 & $\sim 2.4$ & $1.7 \times 10^{-12}$ & 720 & $\sim 2 \times 10^{15}$ & $3.4 \times 10^{3}$ & 1000 \\
\hline Ratios & (see text) & & 205 & $1 / 6$ & 25 & 5000 & $1 / 5000$ \\
\hline
\end{tabular}

identify atomic helium emission at $587.6 \mathrm{~nm}$ and $706.5 \mathrm{~nm}$. The intense helium emission line often observed at $501.6 \mathrm{~nm}$, however, is not detected in this air plasma. Spectral overlap between the $\mathrm{He}$ I line at $587.6 \mathrm{~nm}$ and the $\Delta v=-3$ band of the $\mathrm{B}^{3} \Pi_{\mathrm{g}}-\mathrm{A}^{3} \Sigma_{\mathrm{u}}^{+}$transition in nitrogen can be resolved through simple subtraction. The interference between the He I emission at $706.5 \mathrm{~nm}$ and the $\Delta v=-1$ band is also resolved, but in both cases some uncertainty in the baseline for emission is introduced. Calibration curves for $\mathrm{He}$ in air show that the $\mathrm{He}$ emission intensity intersects the detector's noise floor of 10 counts at a detection limit of DL $\approx 1000 \mathrm{ppm}$. The same microplasma reactor has produced sulfur dioxide detection limits as low as $0.2 \mathrm{ppm}$ using argon as the carrier gas. ${ }^{19}$ The causes for poor DL performance in air are examined next.

The emission of photons from an analyte depends on the generation rate of excited states. Specifically, the number of photons per second produced by the analyte is proportional to the excitation rate constant $\left(k_{\mathrm{ex}}\right)$ and the electron density $\left(n_{\mathrm{e}}\right)$. It is necessary to increase the photon emission above the noise limit of the detector in order to reliably use AES. This can be accomplished by increasing the electron density of the plasma, or increasing the excitation rate constant by increasing the electron temperature. Table 1 shows the estimation of emission intensity for the detection of $\mathrm{SO}_{2}$ in argon and the measurement of $\mathrm{He}$ in room air. The electron temperature in the plasma has been previously reported as a function of pressure for this reactor. ${ }^{23}$ From this electron temperature, the excitation rate constants for $\mathrm{SO}_{2}{ }^{24}$ and $\mathrm{He}^{25}$ are determined. Clearly, the large threshold energy for excitation of $\mathrm{He}$ $(\sim 20 \mathrm{eV})$ compared to $\mathrm{SO}_{2}(5.5 \mathrm{eV})$ results in a decrease in $\mathrm{He}$ excitation frequency. The excitation of helium is further reduced due to the low electron density produced by the air plasma, as compared with argon. Although the input power to each plasma is the same $(2.5 \mathrm{~W})$, the power supplied to the air plasma is more strongly partitioned into non-ionizing collisions due to the molecular nature of the gas. Power loss to non-ionizing collisions is described by the shaded blocks in Fig. 1 and includes dissociation and excitation of translational, vibrational and rotational molecular states. The total energy required for the production of an ion $\left(E_{\mathrm{c}}\right)$ can be calculated from the electron temperature and the collision rate constants for oxygen, ${ }^{26}$ nitrogen, ${ }^{27}$ and argon. ${ }^{26}$ Nitrogen is used as an approximation for air to determine $E_{\mathrm{c}}$ in Table 1 where it is shown that the ionization efficiency is six times lower in nitrogen than argon. The electron density in argon has been measured by Langmuir probe. ${ }^{23}$ To estimate the electron density in air, we have simply scaled the data for argon by the ratio of $E_{\mathrm{c}}(\mathrm{Ar}) / E_{\mathrm{c}}\left(\mathrm{N}_{2}\right)$. In addition, measurements have shown that the plasma generator is approximately four times less efficient in coupling power to the plasma as the pressure is reduced to from 300 to $30 \mathrm{~Pa}^{23}$ This generator inefficiency causes heating of the microstructure and a reduction of power absorbed by the plasma's electrons, as shown in Fig. 1. The estimated electron density in air is therefore reduced by an additional factor of 4 in Table 1. Finally, the emission frequency of photons from the analyte atom or molecule is calculated from $k_{\mathrm{ex}} n_{\mathrm{e}}$. Although the data of Table 1 contain several approximations, the ratios of calculated excitation rates and experimental DLs are in good agreement, demonstrating that the DL for $\mathrm{He}$ in air is reduced by a factor of 5000 due to decreased electron density and increased excitation threshold.

\subsection{Low pressure plasma-on-a-chip: electron cyclotron resonance}

Establishing a high electron density is critical to developing microplasmas with useful detection limits. The electron density has been shown to decrease at low pressure, however, resulting in poor DL performance from microplasmas used for vacuum process monitoring. ${ }^{23}$ In large-scale plasmas, the addition of a magnetic field improves the electron density and emission intensity by using electron cyclotron resonance (ECR) to more efficiently transfer power to the plasma electrons. The effect of an axial magnetic field on the emission intensity of $\mathrm{Ar}$ $(750.4 \mathrm{~nm})$ is presented in Fig. 5 for a miniature ICP operating at $2 \mathrm{~W}$. For the lowest magnet currents $(I<1.3 \mathrm{~A})$, the $B$-field that permeates the plasma is less than required for ECR. Here, there is only a small increase in emission intensity due to the radial confinement of electrons by the axial $B$-field. Once the minimum field strength is established $(I>1.3 \mathrm{~A})$, the cyclotron motion of the plasma electrons is synchronized with the $220 \mathrm{MHz}$ electric field induced by the ICP coil. This efficient means of coupling power to the electrons results in an increase in electron density and emission intensity.

The gradual increase in emission intensity as a function of electromagnet current $(I)$ in Fig. 5 is due to the non-uniform magnetic field strength along the axis of the plasma. At a distance of $z=20 \mathrm{~mm}$ from the $\mathrm{mICP}$, the axial magnetic field strength decays to $B_{z}=32 \mathrm{I}$, from $B_{z}=60 I$ at $z=0$. The emission intensity at $2.7 \mathrm{~Pa}$ is plotted in Fig. 6 as a function of $B_{z}$. Each data point is represented by the minimum and maximum field strength within the plasma. From Fig. 6 it is observed that the emission intensity begins to increase once the threshold of 78 gauss is reached at the mICP window $(z=0)$. The intensity continues to increase until the entire length of the plasma resides within a zone where $B_{z}>78$ gauss. Once the threshold field is exceeded throughout the plasma, the emission intensity saturates. The application of a magnetic field not only increases the emission intensity of the plasma, it is necessary for

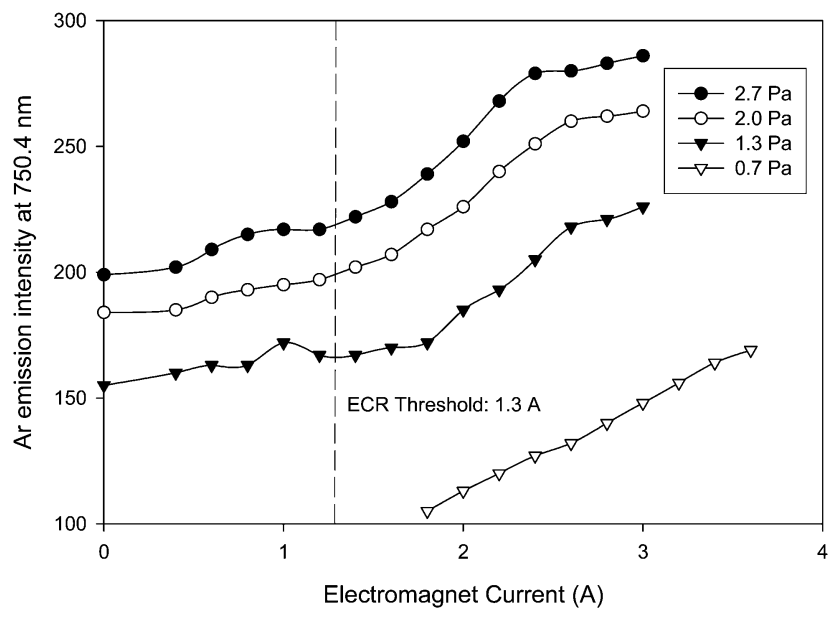

Fig. 5 An enhancement of plasma emission occurs once the electromagnet current exceeds 1.3 A. This corresponds to a peak magnetic field of 78 gauss, which is the condition required for electron cyclotron resonance within this $220 \mathrm{MHz} \mathrm{mICP}$ 


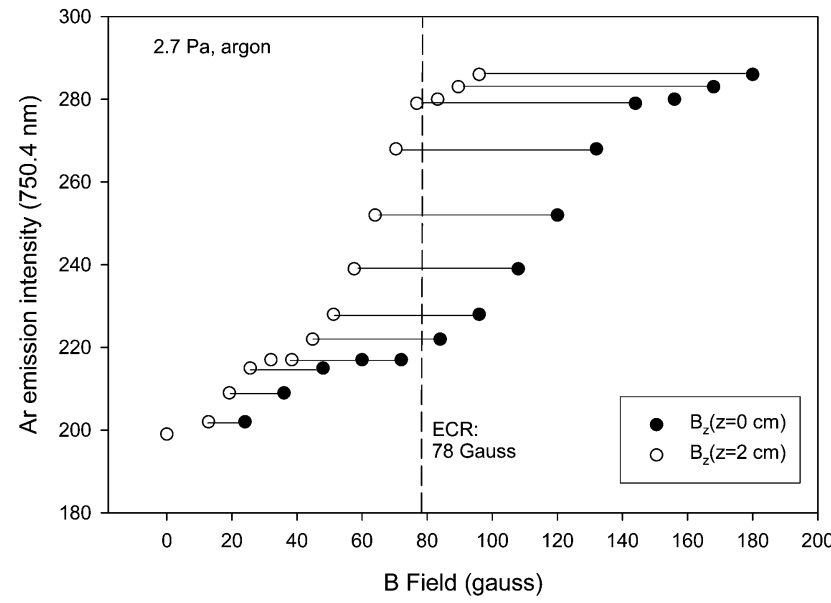

Fig. 6 The gradual enhancement of the plasma emission by ECR is due to the non-uniform static magnetic field within the first $2 \mathrm{~cm}$ of the plasma.

stable discharge operation at the low pressures commonly used by industrial vacuum processes. In particular, the plasma may be used even if $p d \ll 100 \mathrm{~Pa} \mathrm{~cm}$. It should be noted, however, that ECR is not an effective power transfer mechanism for high pressure plasmas. ECR relies on nearly collisionless electron orbits, and therefore the electron collision frequency must be much less than the power supply frequency (i.e., $p \ll 1 / 4 f$ ), as described above.

\subsection{Operation in air at atmospheric pressure}

For many applications of chemical analysis on a chip, an atmospheric pressure microplasma is preferred because complex and expensive vacuum systems are avoided. Although there are many reports of atmospheric pressure microplasmas that function in inert gases, operating in atmospheric pressure air is elusive. The difficulty with the air discharge is the poor ionization efficiency, as outlined in Fig. 1. Most of the energy supplied to the plasma is dissipated in non-ionizing collisions. Fig. 7 shows the result of a calculation that illustrates this point. ${ }^{26,27}$ The total collisional energy consumed in creating a single electron-ion pair is plotted for both argon and nitrogen gas. At atmospheric pressure, the electron temperature is approximately $1 \mathrm{eV}$. From Fig. 7 one may see that the total energy needed to create an argon ion is $E_{\mathrm{c}}\left(T_{\mathrm{e}}=1 \mathrm{eV}\right)=2000 \mathrm{eV}$, but the energy required for nitrogen ionization is the order of $60 \mathrm{keV}$ per ion, for the same electron temperature. The excess energy is partitioned into nitrogen dissociation, excitation of molecular states, and elastic collisions that increase the gas

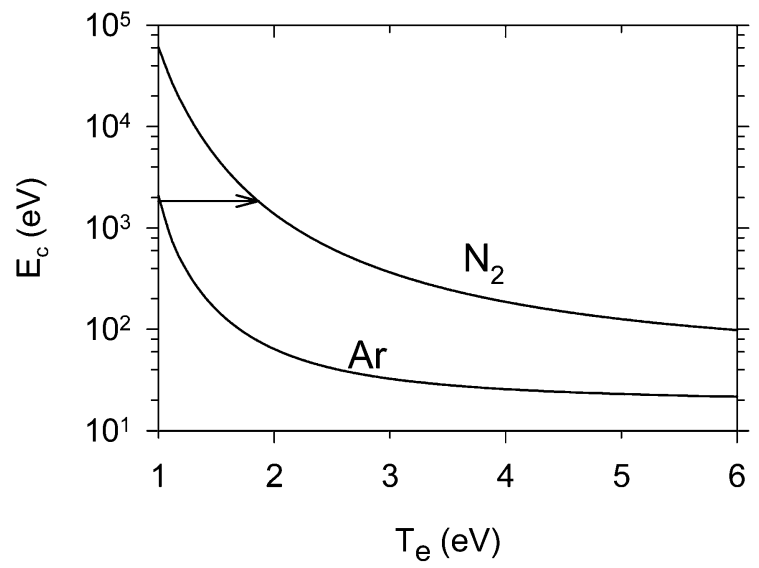

Fig. 7 The total collisional energy required per electron-ion pair $\left(E_{\mathrm{c}}\right)$ is shown as a function of the electron temperature in both argon and nitrogen plasmas. temperature. In order to sustain a nitrogen discharge, more than an order of magnitude more power is required as compared with an identical argon discharge. In air, oxygen also contributes to the problem of poor ionization efficiency ${ }^{26}$ due to electron attachment.

The microplasma generator shown in Fig. 3 is capable of initiating and sustaining an atmospheric air plasma using $3 \mathrm{~W}$ of power or less. The key to the operation of the split-ring resonator is the micromachined $25 \mu \mathrm{m}$ discharge gap. The gap concentrates the available power into a plasma volume of the order of $10^{-7} \mathrm{~cm}^{3}$. The power density in a $1 \mathrm{~W}$ discharge is therefore $\sim 10^{7} \mathrm{~W} \mathrm{~cm}^{-3}$, which is many orders of magnitude higher than common glow discharge plasmas $\left(\sim 10 \mathrm{~W}-\mathrm{cm}^{-3}\right)$. At such a high power density, sufficient ionization power is available to sustain the discharge in nitrogen and oxygen in spite of the poor ionization efficiency. Despite the rather high power density, the gas temperature of the split-ring resonator microplasma is the order of $400 \mathrm{~K}$ in argonnitrogen mixtures at one atmosphere. ${ }^{28}$ The low temperature of this non-equilibrium microdischarge makes it possible to operate the device without damage to the delicate microstructured electrodes, and eliminates the need for heat sinks. ${ }^{1}$

The other important parameter in atmospheric air plasmas is the electron temperature. By referring to Fig. 7, one may observe that a nitrogen plasma operating with an electron temperature of $2 \mathrm{eV}$ will have the same ionization efficiency as an argon plasma with $T_{\mathrm{e}}=1 \mathrm{eV}$, as marked by the arrow. As is shown next, the intense electric field created in the gap region will accelerate the plasma electrons to energies above $1 \mathrm{eV}$. Finite element modeling of the split-ring resonator ${ }^{17}$ shows that the peak voltage across the gap is $V_{\mathrm{g}}=30 \mathrm{~V}$. An electron that is accelerated across this gap will therefore gain $30 \mathrm{eV}$ of energy, except that the electron will collide with gas molecules in transit. The electron mean free path is determined from $\lambda_{\mathrm{e}}=1 / \sigma n_{\mathrm{g}}$, where the collision cross section, $\sigma$, is $\sim 2 \times$ $10^{-20} \mathrm{~m}^{2}$ and the room-temperature gas density is $n_{\mathrm{g}}=2.7 \times$ $10^{25} \mathrm{~m}^{-3}$. At $1 \mathrm{~atm}$, the electron mean free path within the gap is approximately $2 \mu \mathrm{m}$, and the energy gained by an electron between collisions is $\delta E=\lambda_{\mathrm{e}}\left(q V_{\mathrm{g}} / 25 \mu \mathrm{m}\right) \approx 2.4 \mathrm{eV}$. The intense electric field and relatively infrequent collisions cause the electron distribution to be non-Maxwellian (i.e., $T_{\mathrm{e}}$ is illdefined), but the high electron energy induced by a narrow gap improves the ionization rate and efficiency, as indicated in Fig. 7, and allows microplasma operation in atmospheric air using $1 \mathrm{~W}$ of power.

\section{Conclusion}

A review of microplasma techniques shows that technical hurdles exist for both low pressure $(<1 \mathrm{~Pa})$ and high pressure (1 atm) operation. At low pressures, the microplasma may be stabilized and enhanced by using a static magnetic field. The magnetic field causes the plasma's electrons to follow helical trajectories, and if the gyrofrequency of the electrons is matched by that of the $\mathrm{rf}$ electric field, the electrons are collisionlessly energized at low pressure through the mechanism of electron cyclotron resonance. At atmospheric pressure, the electron temperature is low due to a high collision frequency. In this case, very few of the electrons have sufficient energy for ionizing collisions and the ionization efficiency is poor. To sustain an air plasma at $1 \mathrm{~atm}$ using only one watt of power, the volume of the microplasma has been reduced by creating the plasma in a $25 \mu \mathrm{m}$ gap between two ultrahigh frequency (UHF) electrodes. The intense electric field in the gap region accelerates electrons to energies where ionization is more likely to occur. Future refinements in the application of these physical principles to microplasma generation will 
produce reliable sources of atomic emission for inexpensive, portable analysis systems.

\section{Acknowledgements}

The authors wish to thank Mr. Peter Grimes for his valuable assistance in the laboratory. This work is supported by the National Science Foundation under Grant No. DMI-0078406 and DMI-0319494.

\section{References}

1 J. Franzke, K. Kunze, M. Miclea and K. Niemax, J. Anal. At. Spectrom., 2003, 18, pp. 802-807.

2 J. C. T. Eijkel, H. Stoeri and A. Manz, Anal. Chem., 1999, 71 2600-2606.

3 J. C. T. Eijkel, H. Stoeri and A. Manz, Anal.Chem., 2000, 72 , 2547-2552.

4 K. E. Johnson, W. Van der Wilpl and V. Karanassios, Proc. SPIE Int. Soc. Opt. Eng., 2001, 4205, 347.

5 W. C. Davis and R. K. Marcus, Spectrochim. Acta,Part B, 2002, 57, 1473-1486.

6 C. G. Wilson and Y. B. Gianchandani, IEEE Trans. Electron Devices, 2002, 49, 2317-2322.

7 R. H. Stark and K. H. Schoenbach, Appl. Phys. Lett., 1999, 74, 3770-3772.

8 P. Kurunczi, J. Lopez, H. Shah and K. Becker, Int. J. Mass Spectrom., 2001, 205, 277-283.

9 M. Miclea, K. Kunze, G. Musa, J. Franzke and K. Niemax, Spectrochim. Acta, Part B, 2001, 56, 37-43.

10 J. P. Boeuf, J. Phys. D: Appl. Phys., 2003, 36, R53-R79.

11 H. Yoshiki and Y. Horiike, Jpn J. Appl. Phys. Part II, 2001, 40, L360-L362.

12 A. Bass, C. Chevalier and M. W. Blades, J. Anal. At. Spectrom., $2001,16$.
13 E. Stoffels, A. J. Flikweert, W. W. Stoffels and G. M. W. Kroesen, Plasma Sources Sci. Technol., 2002, 11, 383-388.

14 F. Iza and J. Hopwood, Plasma Sources Sci. Technol., 2002, 11, 229-235.

15 A. M. Bilgic, U. Engel, E. Voges, M. Kuckelheim and J. A. C. Broekaert, Plasma Sources Sci. Technol., 2000, 9(1), 1-4.

16 A. M. Bilgic, E. Voges, U. Engel and J. A. C. Broekaert, J. Anal. At. Spectrom., 2000, 15(6), 579-580.

17 F. Iza and J. Hopwood, IEEE Trans. Plasma Sci., 2003, 31(4), $782-787$.

18 J. Hopwood and T. Mantei, J. Vacuum Sci. Technol. A, 2003, 21(5), S139-44.

19 O. Minayeva and J. Hopwood, J. Anal. At. Spectrom., 2002, 17(9), 1103-1107.

20 Y. Yin, J. Messier and J. Hopwood, IEEE Trans. Plasma Sci, 1999, 27(5), 1516-24.

21 T. Ichiki, T. Koidesawa and Y. Horiike, Proceedings of the 3rd International Workshop on Basic Aspects of Non-equilibrium Plasma Interacting with Surfaces (BANPIS), Hyogo, Japan, February 7-8, 2003.

22 F. Iza and J. Hopwood, IEEE Trans. Plasma Sci., 2004, 32(2).

23 O. Minayeva and J. Hopwood, J. Appl. Phys., 2003, 94(5), 28212828.

24 M. Hayashi in Swarm Studies and Inelastic Electron-Molecule Collisions, eds. L.C. Pitchford, B.V. McKoy, A. Chutjian and S. Trajmar, Springer-Verlag, New York, 1987.

25 S. C. Brown, Basic Data of Plasma Physics, MIT Press, Cambridge, USA, 1966, pp. 136-138.

26 M. A. Lieberman and A. J. Lichtenberg, Principles of Plasma Discharges and Materials Processing, Wiley, New York, 1994, p. 81

27 K. Tao, D. Mao and J. Hopwood, J. Appl. Phys., 2002, 91(7), 4040-4048.

28 F. Iza and J. Hopwood, IEEE Trans. Plasma Sci., 2004, 32, 498 504 